\title{
A STUDY OF MOBILE DEVICE PRIVACY CONCERNS AND MOBILE DEVICE SAFETY AWARENESS
}

\author{
Scott Spangler, Middle Georgia State University, scott.spangler@mga.edu
}

\begin{abstract}
The purpose of this study included two parts. Part one attempted to empirically validate an instrument that explains mobile device privacy concerns and mobile device safety awareness. Part two investigates differences between the independent variables of gender, age, and college status) and the dependent variables of mobile device privacy concerns and mobile device safety awareness separately. Data were collected from a convenience sample of 147 college students using mobile devices and analyzed and analyzed using exploratory factor analysis and ANOVA. The results are presented and discussed. Study limitations and recommendations for future research round out the paper.
\end{abstract}

Keywords: mobile devices, privacy, privacy concerns, safety awareness

\section{INTRODUCTION}

Computing devices have evolved into indispensable personal social and organizational platforms in society. The overabundance of personal network devices surged in society over the past decade through scalability, portability, and affordability. Mobile devices, such as laptops, Smartphones, iPads, or tables, have become omnipresent in society. Within this mobile device acceptability construct, a presumed increase in user privacy and safety behaviors can be propagated. This paper seeks to paint a preliminary picture of users' mobile (smart) device privacy concerns and mobile device safety awareness. To define the term "mobile (Smart) devices" the researcher utilizes Traxler's (2016) construction:

Smart-phones, games consoles, digital cameras, media players, netbooks, in-car satellite navigation and handheld computers. Almost every student owns one and uses one, often more than one. Not only do they own them and use them, but they also invest considerable time, effort and resources [in] choosing, buying, customizing and exploiting them. These devices express part or much of their owners' values, affiliations, identity and individuality through their choice and their use. They are both pervasive and ubiquitous, both conspicuous and unobtrusive, both noteworthy and taken-for-granted in the lives of most - but not all students (p. 149).

Mobile (smart) devices have become omnipresent in society and especially in higher education domains. Mobile (smart) devices consist of a plethora of innovations (such as smartphones, tablets, iPads or touch-based innovations), which have increased privacy, safety, and security risks to users. The exponential growth in device consumption can be attributed to reduced costs and societies' behavioral expectations of multitasking, knowledge attainment, and social media connectivity. The reduced cost multifunctioning microcomputers have powerful storage capacities that accommodate users' functionality. Additionally, society's unrestrictive device norms and dogmas have increased users' acceptability and compliance towards habitual and continuous connection on open access networks. The obsession for continuous connectivity can directly associate the culture's increase in vulnerable unauthorized access and privacy and safety risk. The culture's obsession with unsecured network connectivity behavior can be attributed to the increase in users' acceptability to "malicious activity, such as identity theft, blackmailing, active data collection, or defamation" (Gkioulos, Wangen, \& Katsikas, 2017, pp. 2-3).

Naïve users' norms are constructed through society. The mobile device culture overall leans towards oversight. The applications on mobile devices lend users (especially young adults) to ease of unmonitored communication, connectivity, and privacy and security concerns (Boyd, 2014; Spangler \& Skovira, 2015). Subsequently, new "smart" technology interfaces and applications lessen users' focus on security concerns and even simplistic measures built into the devices (Kamber 2017, p. 3). As an example, users' countermeasure security negligence includes "pin" 


\section{Issues in Information Systems \\ Volume 19, Issue 4, pp. 206-215, 2018}

construction casualness. Researchers have observed users' neglecting to update or change (below 50\%) pin numbers for privacy and safety measures (Kurkovsky \& Syta, 2010). Negligent laissez-faire users' attitudes and awareness with smartphone privacy and safety measures overall negate manufactured installed security countermeasures (Parker, Ophoff, Belle, \& Karia, 2015; Wang \& Xing, 2018).

The literature's rhetoric propagates users' sophistication in using technology, innovative knowledge about digital privacy concerns (Boyd, 2014). College level and educational training have been directly correlated with privacy protections (Chen and Chen, 2015). Specifically, mobile device privacy has a direct correlation to users' sophistication with technology's security countermeasures. Privacy and safety concerns are influenced by users' efficacy and ability to manage mobile device privacy countermeasures (p. 16). Particularly, users have a negligent behavior or attitude towards exploring security and privacy risks when downloading mobile device applications and software (Mylonas, Kastania, and Gritzalis, 2013).

Privacy and safety countermeasures on mobile devices are instrumental in protecting data and countering insurgents. Despite the amount of developer applied device countermeasures and actionable training to consumers, users continuously demonstrate privacy and safety negligence (Kurkovsky \& Syta, 2010). Despite users' awareness of mobile device threats to security and privacy, few users take measurable strides to protect their devices from unauthorized access. This includes sheltering and protecting the devices from strong password constructions or protecting the limited constructions from vulnerabilities (Whitty, Doodson, Creese, \& Hodge, 2015). However resilient the privacy and safety countermeasure knowledge bases are constructed, users have continued to demonstrate careless behaviors formed through naïve trust levels in connected smart devices (Ophoff \& Robinson, 2014). The users' confidence in new technologies leads to security, privacy and behavioral issues (Gkioulos et al. 2017, p. 11). Additionally, Wang and Xing (2018) stated a link between users' acceptability and increased privacy and safety risks.

\section{PURPOSE OF THE STUDY}

The purpose of this study was two-fold. First, to empirically validate an instrument that describes mobile device privacy concerns and mobile device safety Awareness. Second, to find out whether there are differences between the independent variables of gender (female and male), age (born after 1980 and born before 1980), and college status (freshman, sophomore, senior, and graduate level) and the dependent variables of Mobile Device Privacy concerns and Mobile Device Safety Awareness separately. Consistent with its purpose the following research questions were stated:

RQ1: Are the two components (mobile device privacy concerns and mobile device safety awareness) of the instrument empirically validated to be reliable and interpretable among their designated items?

RQ2: Are there significant gender differences between mobile device privacy concerns and mobile device safety awareness separately?

RQ3: Are there significant age differences between mobile device privacy concerns and mobile device safety awareness separately?

RQ4: Are there significant college status differences between mobile device privacy concerns and mobile device safety awareness separately?

For the purpose of the present study, the following dependent variables are defined:

Mobile Device Privacy Concerns: Defined as concerns users express about their privacy when using their mobile devices. These concerns include 1) protection of personal information 2) unauthorized access of personal information, 3) control over personal information, and 4) collection of personal information.

Mobile Device Safety Awareness: Defined as awareness and compliance of safety issues when using mobile devices. These safety issues include 1) sharing information with people, 2) taking security measures to avoid access by unauthorized persons, 3) avoiding unauthorized access with password protection, and 4) deploying and updating security software. 


\section{METHODOLOGY}

\section{Instrument}

A two-part Likert-type instrument was used for the present study. Part 1 includes four items that describe mobile device privacy concerns. The items for this part of the instrument were adapted from a study conducted by Koohang (2017). Part 2 consists of four items that describe mobile device safety awareness. This researcher developed the items for this part of the instrument.

The items of the instrument for part 1 and part 2 are as follows:

\section{Part 1 - Mobile Device Privacy Concerns}

1. Protection -- I am concerned that my personal information and data are not protected from unauthorized access when using my mobile device (smart devices).

2. Unauthorized access -- I am concerned that unauthorized people may access my personal information and data when using my mobile device (smart devices).

3. Control -- When performing a task on my mobile device (smart devices), I am concerned that I may not have control over my personal information and data.

4. Collection -- Even when it is necessary to give out information about myself while using an app on my mobile device (smart devices), I am concerned about how my information is collected, processed, and used.

\section{Part 2 - Mobile Device Safety Awareness}

1. Sharing Information -- When I use my mobile device, I am always careful as to what I share with others.

2. Security -- I always make sure that my mobile device is securely locked a few seconds after use.

3. Password protected -- My mobile device is password protected.

4. Threat -- I always make sure that I have the most recent security software updates that protect my mobile device against viruses, malware or other online threats.

The instrument used the following scoring strategy: $7=$ completely agree, $6=$ mostly agree, $5=$ somewhat agree, 4 $=$ neither agree nor disagree, $3=$ somewhat disagree, $2=$ mostly disagree, $1=$ completely disagree.

\section{Sample and Procedure}

Upon approval from the Middle Georgia State University's Internal Review Board (IRB), the instrument was administered to a convenience sample of approximately 600 undergraduate and graduate online information technology students in a medium-sized university in the Southeast part of the United States. A total of 159 students completed the survey. Of the 159, 12 were eliminated because of incomplete data. Of the population, males (70\%) responded with females (30\%) and overall a large number (76\%) indicating that they were born after 1980.

\section{Data analysis}

For RQ1, exploratory factor analysis was conducted to analyze the collected data. The analysis includes KMO and Bartlett's test, test of variance explained, Scree plot, and finally principal component analysis with Varimax rotation showing components that are retained. One-way ANOVAs were conducted to analyze the collected data for RQs 2 , 3 , and 4. The ANOVA test reveals the effect that one independent variable on one dependent variable. ANOVA uses F test to determine the significance of the groups. Groups with more than two levels include Post-hoc analysis. Descriptive analyses show the means and standard deviation of each dependent variable with independent variables. SPSSTM $^{\text {version }} 25$ was used to analyze the collected data.

\section{RESULTS}

First, descriptive analyses are presented to give an overview of the means and standard deviation (with graphical presentation) of all the items for the Mobile Device Privacy Concerns construct and Mobile Device Safety Awareness construct. Second, results for RQ1 are presented, which includes the empirical validation of the instrument. Third, results for RQ2 - RQ4, which includes differences between the independent variables of gender, age, and college 


\section{Issues in Information Systems}

Volume 19, Issue 4, pp. 206-215, 2018

status and the dependent variables of mobile device privacy concerns and mobile device safety awareness separately.

\section{Descriptive Analyses}

The results of descriptive analyses are shown in Table 1. As can be seen, the subjects express high concern about their privacy when using a mobile device. Likewise, the subjects showed a great deal of understanding about safety awareness when using a mobile device. Figures 1 and 2 show the graphical presentation of the results of descriptive analyses.

Table 1. Descriptive Statistics $(\mathrm{N}=147)$

\begin{tabular}{|l|r|r|}
\hline & Mean & $\begin{array}{c}\text { Std. } \\
\text { Deviation }\end{array}$ \\
\hline Privacy: Protection & 5.29 & 1.579 \\
\hline Privacy: Unauthorized access & 5.12 & 1.684 \\
\hline Privacy: Control & 5.14 & 1.588 \\
\hline Privacy: Collection & 6.07 & 1.234 \\
\hline Safety: Sharing information & 6.46 & .846 \\
\hline Safety: Security & 6.35 & 1.403 \\
\hline Safety: Password protected & 6.44 & 1.526 \\
\hline Safety: Threats & 6.41 & 1.071 \\
\hline
\end{tabular}

\section{Privacy Concerns}

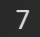

7

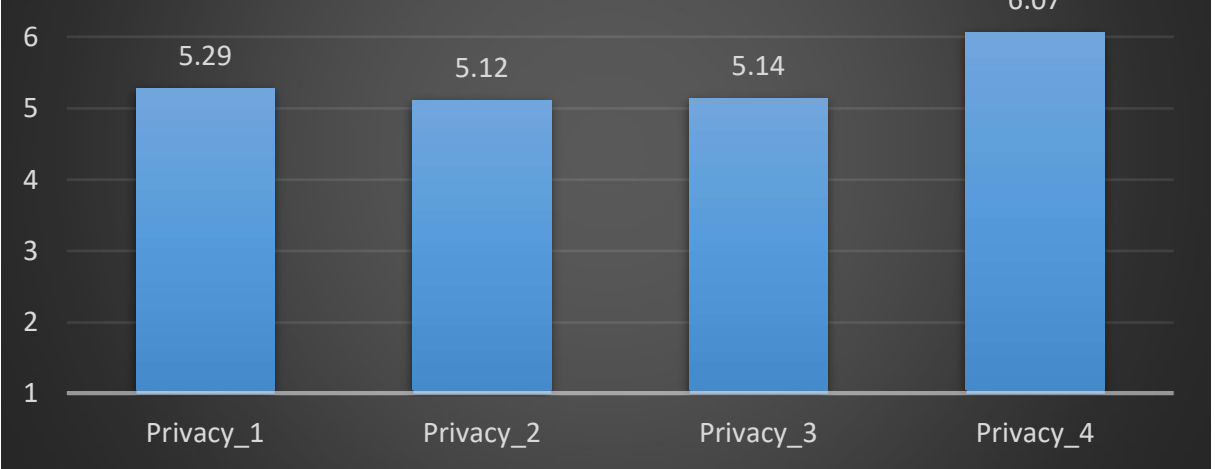

Figure 1. Mobile Device Privacy 


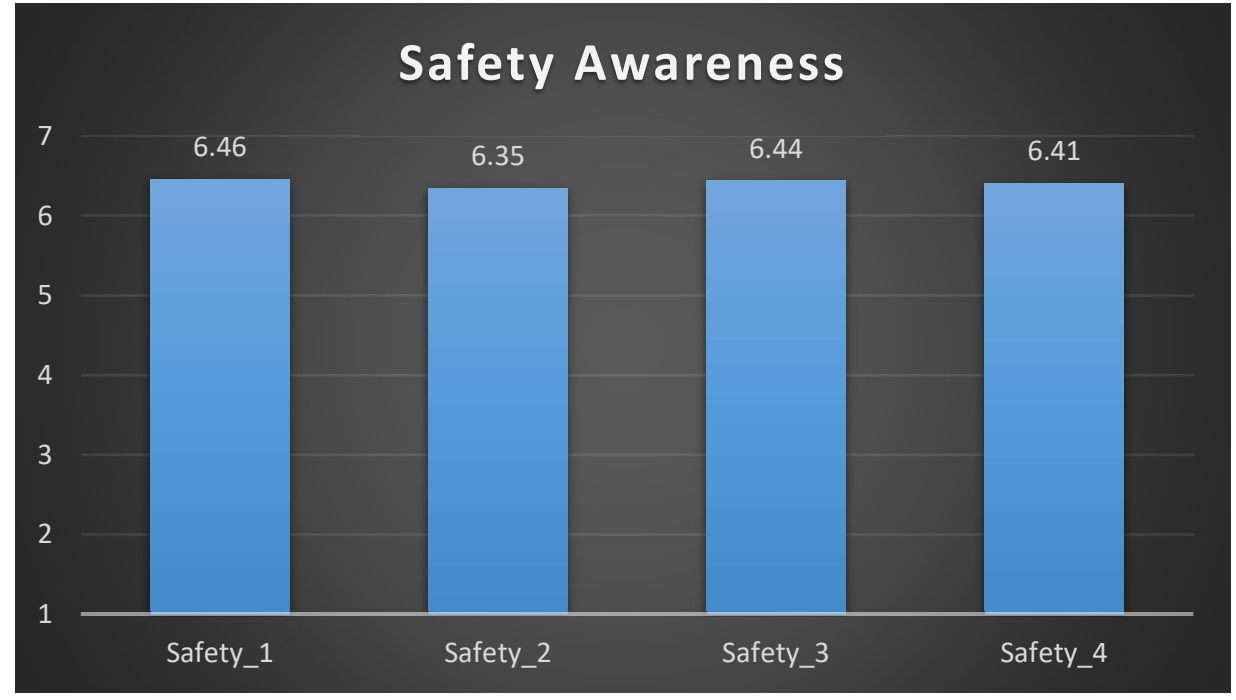

Figure 2. Mobile Device Safety Awareness

RQ1: Are the two components (mobile device privacy concerns and mobile device safety awareness) of the instrument empirically validated to be reliable and interpretable among their designated items?

Table 2 shows the results of KMO and Bartlett's test of sphericity. This test is performed to determine multivariate normality and sampling adequacy. The index for KMO was .739, the Bartlett's test of sphericity was (Chi-Squared = $345.921, \mathrm{df}=28$, and $\mathrm{p}=.000)$. These results indicated the multivariate normality and sampling adequacy.

Table 2. KMO and Bartlett's Test

\begin{tabular}{|l|l|r|}
\hline Kaiser-Meyer-Olkin Measure of Sampling Adequacy. & .739 \\
\hline Bartlett's Test of & Approx. Chi-Square & 345.921 \\
\cline { 2 - 3 } Sphericity & Df & 28 \\
\cline { 2 - 3 } & Sig. & .000 \\
\hline
\end{tabular}

Table 3 shows the test of variance explained for the two retained components (initial eigenvalues, extraction sums of squared loadings, and the rotation sums of squared loadings). The total variance-retain accounted for was $93.61 \%$, which is considered a very high variance. Figure 3 shows the Scree plot, which is a graphical representation of the eigenvalues that uses Principal Component Analysis. As can be seen, the plot has detected two components - this is a further validation of the number of components retained.

Table 3. Total Variance Explained

\begin{tabular}{|l|c|r|r|}
\hline \multirow{2}{*}{ Component } & \multicolumn{3}{|c|}{ Initial Eigenvalues } \\
\cline { 2 - 4 } & Total & \% of Variance & Cumulative \% \\
\hline 1 & 2.767 & 34.593 & 34.593 \\
\hline 2 & 2.006 & 25.078 & 59.672 \\
\hline \multirow{2}{*}{ Component } & \multicolumn{2}{|c|}{ Extraction Sums of Squared Loadings } \\
\cline { 2 - 4 } & Total & \% of Variance & Cumulative \% \\
\hline 1 & 2.767 & 34.593 & 34.593 \\
\hline 2 & 2.006 & 25.078 & 59.672 \\
\hline \multirow{2}{*}{ Component } & \multicolumn{3}{|c|}{ Rotation Sums of Squared Loadings } \\
\cline { 2 - 4 } & Total & \% of Variance & Cumulative \% \\
\hline 1 & 2.731 & \multicolumn{3}{|c|}{34.138} & 34.138 \\
\hline 2 & 2.043 & 25.534 & 59.672 \\
\hline
\end{tabular}

Extraction Method: Principal Component Analysis. 


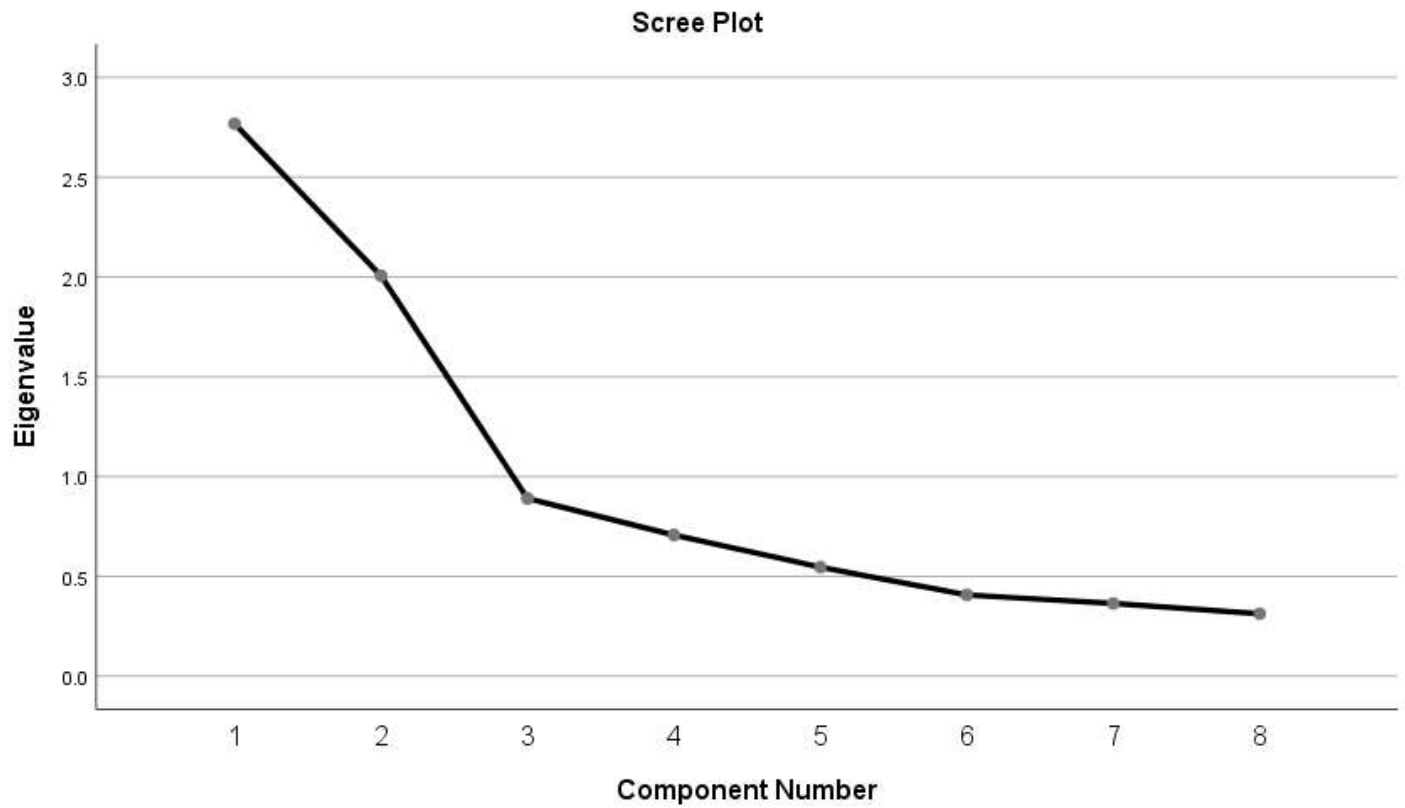

Figure 3. Scree Plot

Table 4 shows the results of the rotated component matrix using varimax rotation. Component 1 retained four items that were designated for the Mobile Device Privacy concerns (Protection, Unauthorized access, Control, and Collection). Component 2 retained four items that were designated for the Mobile Device Safety Awareness (Sharing information, Security, Password protected, and Threats). The results of the reliability tests for Mobile Device Privacy concerns and Mobile Device Safety Awareness were .83 and .67 respectively.

Table 4. Rotated Component Matrix

\begin{tabular}{|l|l|c|r|}
\hline \multirow{4}{*}{ Mobile Device Privacy } & Items/Factors & \multicolumn{2}{|c|}{ Component } \\
\cline { 2 - 4 } & & 1 & 2 \\
\cline { 2 - 4 } & Privacy: Protection & $\mathbf{. 8 1 2}$ & .071 \\
\cline { 2 - 4 } & Privacy: Unauthorized access & $\mathbf{. 8 6 3}$ & .043 \\
\cline { 2 - 4 } & Privacy: Control & $\mathbf{. 8 3 6}$ & -.061 \\
\cline { 2 - 4 } & Privacy: Collection & $\mathbf{. 7 5 8}$ & .072 \\
\hline \multirow{3}{*}{$\begin{array}{l}\text { Mobile Device Safety } \\
\text { Awareness }\end{array}$} & Safety: Sharing information & .203 & $\mathbf{. 5 4 8}$ \\
\cline { 2 - 4 } & Safety: Security & -.017 & $\mathbf{. 8 5 8}$ \\
\cline { 2 - 4 } & Safety: Password protected & .041 & $\mathbf{. 7 4 6}$ \\
\cline { 2 - 4 } & Safety: Threats & -.097 & $\mathbf{. 6 5 9}$ \\
\hline
\end{tabular}

Extraction Method: Principal Component Analysis. Rotation Method: Varimax with Kaiser Normalization.

RQ2: Are there significant gender differences between mobile device privacy concerns and mobile device safety awareness separately?

The results of ANOVA for RQ2 revealed a significant mean difference at exactly .05 alpha level of significance $\left(F_{1}\right.$, $\left.{ }_{145}=3.873, .050\right)$. Male subjects appeared to have more concern about their privacy when using mobile devices. There was no significant mean difference between gender and subjects' safety awareness regarding mobile devices. Females and males were equally and exceedingly aware of mobile devices safety issues and complying with the safety measures. Tables 5 shows the results of ANOVA. descriptive analysis is shown in Table 6 . 


\section{Issues in Information Systems}

Volume 19, Issue 4, pp. 206-215, 2018

Table 5. One-way ANOVA for Gender $(\mathrm{N}=147)$

\begin{tabular}{|l|l|r|r|r|r|r|}
\hline \multicolumn{2}{|c|}{} & $\begin{array}{l}\text { Sum of } \\
\text { Squares }\end{array}$ & df & $\begin{array}{l}\text { Mean } \\
\text { Square }\end{array}$ & F & Sig. \\
\hline \multirow{4}{*}{ Mobile Device Privacy } & Between Groups & 5.971 & 1 & 5.971 & 3.873 & .050 \\
\cline { 2 - 7 } & Within Groups & 223.555 & 145 & 1.542 & & \\
\cline { 2 - 7 } & Total & 229.526 & 146 & & & \\
\hline \multirow{2}{*}{$\begin{array}{l}\text { Mobile Device Safety } \\
\text { Awareness }\end{array}$} & Between Groups & .103 & 1 & .103 & .132 & .717 \\
\cline { 2 - 7 } & Within Groups & 113.229 & 145 & .781 & & \\
\cline { 2 - 7 } & Total & 113.332 & 146 & & & \\
\hline
\end{tabular}

Table 6. Descriptive Analysis for Gender $(\mathrm{N}=147)$

\begin{tabular}{|l|l|r|r|r|}
\hline \multicolumn{2}{|c|}{} & N & Mean & $\begin{array}{l}\text { Std. } \\
\text { Deviation }\end{array}$ \\
\hline \multirow{3}{*}{ Mobile Device Privacy } & Female & 43 & 5.0930 & 1.27478 \\
\cline { 2 - 5 } & Male & 104 & 5.5361 & 1.22792 \\
\cline { 2 - 5 } & Total & 147 & 5.4065 & 1.25383 \\
\hline \multirow{2}{*}{$\begin{array}{l}\text { Mobile Device Safety } \\
\text { Awareness }\end{array}$} & Female & 43 & 6.3721 & 1.02407 \\
\cline { 2 - 5 } & Male & 104 & 6.4303 & .81956 \\
\cline { 2 - 5 } & Total & 147 & 6.4133 & .88105 \\
\hline
\end{tabular}

RQ3: Are there significant age differences between mobile device privacy concerns and mobile device safety awareness separately?

The results of ANOVA for RQ3 revealed no significant mean difference between age (subjects born before 1980, known as digital immigrants and subjects born after 1980, known as digital natives) and the mobile devices privacy concerns and mobile devices safety awareness. Though not significant, both age groups were equally concerned about their privacy, furthermore, they were equally and exceedingly aware of mobile devices safety issues and complying with the safety measures. Tables 7 shows the results of ANOVA. Descriptive analysis is shown in Table 8.

Table 7. One-way ANOVA for Age $(\mathrm{N}=147)$

\begin{tabular}{|l|l|r|r|r|r|r|}
\hline \multicolumn{2}{|c|}{} & $\begin{array}{l}\text { Sum of } \\
\text { Squares }\end{array}$ & df & $\begin{array}{l}\text { Mean } \\
\text { Square }\end{array}$ & F & Sig. \\
\hline \multirow{4}{*}{ Mobile Device Privacy } & Between Groups & .130 & 1 & .130 & .082 & .774 \\
\cline { 2 - 8 } & Within Groups & 229.396 & 145 & 1.582 & & \\
\cline { 2 - 8 } & Total & 229.526 & 146 & & & \\
\hline \multirow{2}{*}{$\begin{array}{l}\text { Mobile Device Safety } \\
\text { Awareness }\end{array}$} & Between Groups & .167 & 1 & .167 & .213 & .645 \\
\cline { 2 - 8 } & Within Groups & 113.165 & 145 & .780 & & \\
\cline { 2 - 7 } & Total & 113.332 & 146 & & & \\
\hline
\end{tabular}

Table 8. Descriptive Analysis for Age $(\mathrm{N}=147)$

\begin{tabular}{|l|l|r|r|r|}
\hline \multicolumn{2}{|c|}{} & N & Mean & \multicolumn{1}{l|}{$\begin{array}{l}\text { Std. } \\
\text { Deviation }\end{array}$} \\
\hline \multirow{3}{*}{ Mobile Device Privacy } & Born after 1980 & 111 & 5.4234 & 1.20736 \\
\cline { 2 - 5 } & Born before 1980 & 36 & 5.3542 & 1.40455 \\
\cline { 2 - 5 } & Total & 147 & 5.4065 & 1.25383 \\
\hline \multirow{2}{*}{$\begin{array}{l}\text { Mobile Device Safety } \\
\text { Awareness }\end{array}$} & Born after 1980 & 111 & 6.4324 & .84608 \\
\cline { 2 - 5 } & Born before 1980 & 36 & 6.3542 & .99171 \\
\cline { 2 - 5 } & Total & 147 & 6.4133 & .88105 \\
\hline
\end{tabular}

RQ4: Are there significant college status differences between mobile device privacy concerns and mobile device safety awareness separately?

The results of ANOVA for RQ4 revealed no significant mean difference between subjects' college level and mobile 


\section{Issues in Information Systems \\ Volume 19, Issue 4, pp. 206-215, 2018}

devices privacy concerns and mobile devices safety awareness. Though not significant, all groups were equally concerned about their privacy. They were, however, a significant difference between the college level and mobile device safety awareness. The higher the college level, the higher mobile device safety awareness. Tables 8 shows the results of ANOVA. Descriptive analysis is shown in Table 9.

Table 9. One-way ANOVA for College Status $(\mathrm{N}=147)$

\begin{tabular}{|l|l|r|r|r|r|r|}
\hline \multicolumn{2}{|c|}{} & $\begin{array}{l}\text { Sum of } \\
\text { Squares }\end{array}$ & df & $\begin{array}{l}\text { Mean } \\
\text { Square }\end{array}$ & F & Sig. \\
\hline \multirow{3}{*}{ Mobile Device Privacy } & Between Groups & 5.033 & 3 & 1.678 & 1.069 & .364 \\
\cline { 2 - 8 } & Within Groups & 224.493 & 143 & 1.570 & & \\
\cline { 2 - 7 } & Total & 229.526 & 146 & & & \\
\hline \multirow{2}{*}{$\begin{array}{l}\text { Mobile Device Safety } \\
\text { Awareness }\end{array}$} & Between Groups & 9.489 & 3 & 3.163 & 4.356 & .006 \\
\cline { 2 - 8 } & Within Groups & 103.843 & 143 & .726 & & \\
\cline { 2 - 8 } & Total & 113.332 & 146 & & & \\
\hline
\end{tabular}

Table 10. Descriptive Analysis for College Status $(\mathrm{N}=147)$

\begin{tabular}{|l|l|r|r|r|}
\hline \multicolumn{2}{|c|}{} & N & \multicolumn{1}{l|}{ Mean } & \multicolumn{1}{l|}{$\begin{array}{l}\text { Std. } \\
\text { Deviation }\end{array}$} \\
\hline \multirow{4}{*}{ Mobile Device Privacy } & Sophomore & 15 & 5.3167 & 1.25167 \\
\cline { 2 - 5 } & Junior & 46 & 5.2880 & 1.34264 \\
\cline { 2 - 5 } & Senior & 65 & 5.6038 & 1.10653 \\
\cline { 2 - 5 } & Graduate Level & 21 & 5.1190 & 1.46761 \\
\cline { 2 - 5 } & Total & 147 & 5.4065 & 1.25383 \\
\hline \multirow{4}{*}{$\begin{array}{l}\text { Awobile Device Safety } \\
\text { Awareness }\end{array}$} & Sophomore & 15 & 5.8167 & 1.32445 \\
\cline { 2 - 5 } & Junior & 46 & 6.2772 & .94167 \\
\cline { 2 - 5 } & Senior & 65 & 6.5423 & .76108 \\
\cline { 2 - 5 } & Graduate Level & 21 & 6.7381 & .33982 \\
\cline { 2 - 5 } & Total & 147 & 6.4133 & .88105 \\
\hline
\end{tabular}

Note: There were no freshmen participated in the study

\section{CONCLUSION}

The purpose of this study was to 1) empirically validate an instrument that describes mobile device privacy concerns and mobile device safety awareness and 2) investigate differences between the independent variables of (gender, age, and college status) and the dependent variables of mobile device privacy concerns and mobile device safety awareness separately. The descriptive analyses showed that subjects expressed high concern about their privacy when using a mobile device. Conversely, the subjects showed an increased understanding of safety awareness when using a mobile device. This implies that while the subjects show increased concern towards privacy, they express increased awareness of and compliance with the safety issues of mobile devices.

The exploratory factor analysis findings revealed an empirically validated instrument with two constructs -- mobile device privacy concerns and mobile device safety awareness. This implies that each construct retained the four items that were designated to it. Therefore, the constructs can measure users' mobile device privacy concerns and mobile device safety awareness.

The ANOVA findings for gender indicated that male subjects had more concern about their privacy when using mobile devices than female subject did. This finding, in general, is not in line with previous literature where women generally appearing more concerned about their privacy (Murnane, 2016; Shaheen, 1999). This may be due to the unequal number of subjects (male and female) used in this study. This finding may also imply that the trend for college students sampled for this study may be different from the general population. There was no significant difference between gender and subjects' mobile device safety awareness regarding mobile devices implying that females and males were equally aware of mobile devices safety issues and were complying with the safety measures. 


\section{Issues in Information Systems \\ Volume 19, Issue 4, pp. 206-215, 2018}

The ANOVA finding for age (subjects born before 1980, known as digital immigrants and subjects born after 1980, known as digital natives) indicated no significant difference for both mobile devices privacy concerns and mobile devices safety awareness. Although there were no significant differences, both digital immigrants and digital natives were equally concerned about their privacy and were equally aware of mobile devices safety issues and complying with the mobile device safety measures.

The results of ANOVA for college status revealed no significant mean difference between subjects' college level and mobile devices privacy concerns and mobile devices safety awareness. Though not significant, all groups were equally concerned about their privacy. They were, however, a significant difference between the college level and mobile device safety awareness. The higher the college level, the higher mobile device safety awareness. This implies that as students mature academically, they become more aware of mobile devices safety issues and complying with the mobile device safety measures.

Additionally, Gkioulos, et al. (2017) research pointed to a significant finding that suggested overconfidence in mobile devices leads to "negligent behaviors" (p. 11). Again, this pilot study reflects a higher consideration towards utilizing security and privacy protocols amongst all users and gender equality on the construction. The pilot did contrast the study uncovering preliminary observations for a higher regard for safety and privacy with users' escalation in academics and knowledge of countermeasures. Also, the study contrasted Kurkovsky \& Syta's (2010) findings that suggest user negligence with privacy and safety considerations and a later study by Whitty, Doodson, Creese, \& Hodge's (2015).

This research contrasts the literature findings in connection with risks. Gkioulos, et al. (2017) summarized users are willing to "accept the risk" in order to gain services. This study contrasts this finding suggesting IT students are highly intuned with privacy and safety concerns countermeasures. As reflected in Table 10, the study demonstrates an escalation in knowledge and concern for safety as students progress through their academic discipline or academic maturity. Additionally, the pilot study observed equal concerns between the genders and age groups about privacy and safety. These two aspects contrast the earlier literature. However, this consideration may be influenced by the large population born after 1980. The finding contrasts Whitty, Doodson, Creese, \& Hodges' (2015) study that stated the participants were fluent in sharing passwords. This contrast may be greater based on the participant's academic constructs. However, this study did concur with Gkioulos's (2017) confidences that information technology students seem informed about privacy and safety protocols. Understanding this consideration, the study must consider its limitations in population and geographical lack in diversity.

To further this research and provide deeper insights, the researchers suggest a larger study to compare and contrast differences between academic domains. It also calls for a larger and more diverse population and less concentrated (geographically) to determine if the findings are generalizable. Additionally, future research may collect participants' qualitative responses, which could shed a greater distinction concerning the participants' reflections on an understanding of countermeasures for privacy and safety considerations of mobile devices.

\section{REFERENCES}

Boyd, D. (2014). It's complicated: The social lives of networked teens. Yale University Press: London.

Chen, H. T., \& Chen, W. (2015). Couldn't or wouldn't? The influence of privacy concerns and self-efficacy in privacy management on privacy protection. Cyberpsychology, Behavior and Social Networking, 18(1), 1319. https://doi.org/10.1089/cyber.2014.0456.

Gkioulos, V., Wangen, G., \& Katsikas, S. K. (2017). User modelling validation over the security awareness of digital natives. Future Internet, 9(3), 32. https://doi.org/10.3390/fi9030032.

Gkioulos, V., Wangen, G., Katsikas, S. K., Kavallieratos, G., \& Kotzanikolaou, P. (2017). Security awareness of the digital natives. Information, 8(2), 42. https://doi.org/10.3390/info8020042.

Kamber, T. (2017). Gen X: The cro-magnon of digital natives. Generations, 4(3), 48-54. 
Koohang, A. (2017). Social media sites privacy concerns: Empirical validation of an instrument. Online Journal of Applied Knowledge Management, 5(1), 14-26.

Kurkovsky, S., \& Syta, E. (2010). Digital natives and mobile phones: A survey of practices and attitudes about privacy and security. In 2010 IEEE International Symposium on Technology and Society (pp. 441-449). https://doi.org/10.1109/ISTAS.2010.5514610.

Murnane, K. (2016). How men and women differ in their approach to online privacy and security. Forbes Magazine. Retrieved July 1, 2018 from https://www.forbes.com/sites/kevinmurnane/2016/04/11/how-men-andwomen-differ-in-their-approach-to-online-privacy-and-security/

Mylonas, A., Kastania, A., \& Gritzalis (2013). Delegate the smartphone users? Security awareness in smartphone platforms. Computer security 34, 47-66.

Ophoff, J., Robinson, M. (2014). Exploring end-user smartphone security awareness within a South African context. In the Proceedings of the Information Security for South Africa, Johannesburg, South Africa.

Parker, F., Ophoff, J., Van Belle, J.P., \& Karia, R. (2015). Security awareness and security adoption of security controls by smartphone users. In the Proceedings of the Information Security for South Africa, Johannesburg, South Africa.

Prensky, M. (2012). From digital natives to digital wisdom: hopeful essays for 21 st century learning. Thousand Oaks, Calif.: Corwin.

Sheehan, K. B. (1999). An investigation of gender differences in on-line privacy concerns and resultant behaviors. Journal of Interactive Marketing, 13(4), 24-38.

Spangler, S. \& Skovira, R. (2015). Digital natives and mobile technology addiction. Issues in Information Systems, 16(1), 1-6.

Traxler, J. (2016). Students and mobile devices, ATL-J. Taylor and Frances, 18(2), 149-160. https://doi.org/10.1080/09687769.2010.492847

Whitty, M., Doodson, J., Creese, S., \& Hodges, D. (2015). Individual differences in cyber security behaviors: an examination of who is sharing passwords. Cyberpsychology, Behavior and Social Networking, 18(1), 3-7. https://doi.org/10.1089/cyber.2014.0179

Wang, X., \& Xing, W. (2018). Exploring the influence of parental involvement and socioeconomic status on teen digital citizenship: A path modeling approach. Journal of Educational Technology \& Society, 21(1), 186199. 